\title{
On the module of effective relations of a standard algebra
}

\author{
By Francesc Planas-Vilanova
}

Dept. Matemàtica Aplicada I. ETSEIB-UPC. Diagonal 647, E-08028 Barcelona.

\section{Introduction}

Let $A$ be a commutative ring. We denote by a standard $A$-algebra a commutative graded $A$-algebra $U=\oplus_{n \geq 0} U_{n}$ with $U_{0}=A$ and such that $U$ is generated as an $A$-algebra by the elements of $U_{1}$. Take $\underline{x}$ a set of (possibly infinite) generators of the $A$-module $U_{1}$. Let $V=A[\underline{t}]$ be the polynomial ring with as many variables $\underline{t}$ (of degree one) as $\underline{x}$ has elements and let $f: V \rightarrow U$ be the graded free presentation of $U$ induced by the $\underline{x}$. For $n \geq 2$, we will call module of effective $n$-relations the $A$-module $E(U)_{n}=\operatorname{ker} f_{n} / V_{1} \cdot \operatorname{ker} f_{n-1}$. The minimum positive integer $r \geq 1$ such that the effective $n$-relations are zero for all $n \geq r+1$ is known to be an invariant of $U$. It is called the relation type of $U$ and is denoted by $\operatorname{rt}(U)$. For an ideal $I$ of $A$, we define $E(I)_{n}=E(\mathcal{R}(I))_{n}$ and $\operatorname{rt}(I)=\operatorname{rt}(\mathcal{R}(I))$, where $\mathcal{R}(I)=\oplus_{n \geq 0} I^{n} t^{n} \subset A[t]$ is the Rees algebra of $I$.

In this paper, we give two descriptions of the $A$-module of effective $n$-relations. In terms of André-Quillen homology we have that $E(U)_{n}=H_{1}(A, U, A)_{n}$ (see 2.3). It turns out that this module does not depend on the chosen $\underline{x}$. In terms of Koszul homology we prove that $E(U)_{n}=H_{1}(\underline{x} ; U)_{n}$ (see 2.4). Using these characterizations, we show later some properties on the module of effective $n$-relations and the relation type of a graded algebra. Meanwhile, our line of disquisition approaches us to several earlier works on the subject (see [2], [5], [6], [7], [9], [10], [13] and [14]).

Section 2 is devoted to state the above mentioned (co)homological characterizations of the $A$-module of effective $n$-relations and compare them with some already known results. In section 3 , we give some applications. The interest is specially centered on the module of $n$-relations of powers of an ideal and the module of $n$-relations of Veronese subrings. In particular, one concludes that $\operatorname{rt}\left(U^{(p)}\right) \leq \operatorname{rt}\left(U_{+}^{p}\right)$ but, in general, $\operatorname{rt}\left(U^{(p)}\right) \neq \operatorname{rt}\left(U_{+}^{p}\right)$, where $U_{+}=\oplus_{n>0} U_{n}$ is the irrelevant ideal of $U$ and $U^{(p)}=\oplus_{n \geq 0} U_{n p}$ is the $p$-th Veronese subring of $U$ (see 3.12). Finally, in section 4 we characterize, in terms of a system of generators, which ideals have module of effective $n$-relations zero. In particular, a new characterization of sequences of linear type is obtained.

e-mail: planas@ma1.upc.es 


\section{Homological description of effective relations}

Let $U=\oplus_{n \geq 0} U_{n}$ be a standard $A$-algebra. Put $U_{+}=\oplus_{n>0} U_{n}$ its irrelevant ideal. If $E=\oplus_{n \geq 1} E_{n}$ is a graded $U$-module and $r \geq 1$, we denote by $F_{r}(E)$ the submodule of $E$ generated by the elements of degree at most $r$. Put (possibly infinite)

$$
\mathrm{s}(E)=\min \left\{r \geq 1 \mid E_{n}=0 \text { for all } n \geq r+1\right\} .
$$

Since $\left(E / U_{+} E\right)_{n}=E_{n} / U_{1} E_{n-1}$, then the following three conditions are equivalent: $F_{r}(E)=$ $E, \mathrm{~s}\left(E / U_{+} E\right) \leq r$, and, $E_{n}=U_{1} E_{n-1}$ for all $n \geq r+1$.

Given $h: W \rightarrow U$, a surjective graded morphism of standard $A$-algebras, we are interested in the graded $A$-module $E(h)=\operatorname{ker} h / W_{+} \cdot \operatorname{ker} h$. The following is an elementary, but useful lemma:

Lemma 2.1 Let $f: V \rightarrow U$ and $g: W \rightarrow V$ be two surjective graded morphisms of standard A-algebras. Then, there exists a graded exact sequence of $A$-modules:

$$
E(g) \rightarrow E(f \circ g) \stackrel{g}{\rightarrow} E(f) \rightarrow 0 .
$$

In particular, $\mathrm{s}(E(f)) \leq \mathrm{s}(E(f \circ g)) \leq \max (\mathrm{s}(E(f)), \mathrm{s}(E(g)))$. Moreover, if $V$ and $W$ are two symmetric algebras, then $E(g)_{n}=0$ and $E(f \circ g)_{n}=E(f)_{n}$ for all $n \geq 2$.

Proof. Exact sequence (1) follows from the snake lemma applied to the commutative diagram:

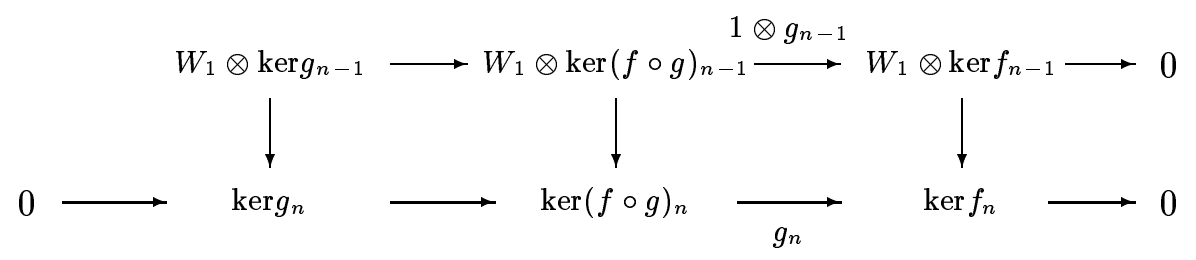

Moreover, if $W=\mathbf{S}\left(W_{1}\right)$ and $V=\mathbf{S}\left(V_{1}\right)$, then $\operatorname{ker} g=F_{1}(\operatorname{ker} g)$

Definition 2.2 Let $U$ be a standard $A$-algebra and let $\alpha: \mathbf{S}\left(U_{1}\right) \rightarrow U$ be the graded morphism of standard $A$-algebras induced by the identity on $U_{1}$. Given $n \geq 2$, the module of effective $n$-relations of $U$ is defined to be $E(U)_{n}=\operatorname{ker} \alpha_{n} / U_{1} \cdot \operatorname{ker} \alpha_{n-1}$. Put $E(U)=$ $\oplus_{n \geq 2} E(U)_{n}=\operatorname{ker} \alpha / \mathbf{S}_{+}\left(U_{1}\right) \cdot \operatorname{ker} \alpha$. Then, the relation type of $U$ is defined to be $\operatorname{rt}(U)=$ $\mathrm{s}(E(U))$. Remark that if $h: W \rightarrow U$ is any symmetric presentation of $U$, that is, $W$ is a symmetric algebra and $h$ is a surjective graded morphism of standard $A$-algebras, then $h$ can be factorized into $h=f \circ g$, where $g: \mathbf{S}\left(W_{1}\right) \rightarrow \mathbf{S}\left(U_{1}\right)$ is the induced morphism by $h_{1}: W_{1} \rightarrow U_{1}$ and $f=\alpha$. Thus, applying Lemma 2.1, $E(U)_{n}=E(h)_{n}$ for all $n \geq 2$ and $\mathrm{s}(E(U))=\mathrm{s}(E(h))$. If $I$ is an ideal of $A$, the module of effective $n$-relations of $I$ is $E(I)_{n}=E(\mathcal{R}(I))_{n}$ and the relation type of $I$ is $\operatorname{rt}(I)=\operatorname{rt}(\mathcal{R}(I))$, where $\mathcal{R}(I)=\oplus_{n \geq 0} I^{n} t^{n}$ is the Rees algebra of $I$. An ideal with module of effective 2-relations zero is called syzygetic. An ideal of relation type 1 is called of linear type (see, e.g., [8]). 
Remark 2.3 In fact, sequence (1) is part of a long exact sequence of André-Quillen homology. Indeed, the Jacobi-Zariski sequence associated to the morphisms $g: W \rightarrow V$ and $f: V \rightarrow U$, with respect to the $U$-module $A=U / U_{+}$, gives rise to

$$
\ldots \rightarrow H_{1}(W, V, A) \rightarrow H_{1}(W, U, A) \rightarrow H_{1}(V, U, A) \rightarrow H_{0}(W, V, A) \rightarrow \ldots
$$

Using $H_{1}(A, A / I, M)=I / I^{2} \otimes M$ and $H_{0}(A, A / I, M)=0$ for any ideal $I$ of $A$ and any $A / I$-module $M$, we get (1) (see [1]).

On the other hand, the Jacobi-Zariski sequence associated to the morphisms $A \rightarrow \mathbf{S}\left(U_{1}\right)$ and $\alpha: \mathbf{S}\left(U_{1}\right) \rightarrow U$, with respect to the $U$-module $A$, is

$$
\ldots \rightarrow H_{1}\left(A, \mathbf{S}\left(U_{1}\right), A\right) \rightarrow H_{1}(A, U, A) \rightarrow H_{1}\left(\mathbf{S}\left(U_{1}\right), U, A\right) \rightarrow H_{0}\left(A, \mathbf{S}\left(U_{1}\right), A\right) \rightarrow \ldots
$$

Using $H_{1}\left(A, \mathbf{S}\left(U_{1}\right), A\right)=0$ and $H_{0}\left(A, \mathbf{S}\left(U_{1}\right), A\right)=H_{0}(A, U, A)$, we get the graded isomorphism of $A$-modules $H_{1}(A, U, A)=H_{1}\left(\mathbf{S}\left(U_{1}\right), U, A\right)=\operatorname{ker} \alpha / \mathbf{S}_{+}\left(U_{1}\right) \cdot \operatorname{ker} \alpha$. Thus, $H_{1}(A, U, A)_{n}=E(U)_{n}$ is the module of effective $n$-relations of $U$. In particular, $\operatorname{rt}(U)=$ $\mathrm{s}\left(H_{1}(A, U, A)\right)$.

There is also a description of the module of effective $n$-relations in terms of Koszul (co)homology. Let $f: V \rightarrow U$ be a surjective graded morphism of standard $A$-algebras. For each $p \geq 1$, consider the map $V_{p} \otimes U \rightarrow U$ sending $x \otimes y$ to $f_{p}(x) y$ and let $\mathcal{K}(f, p)$ be the Koszul complex associated to this $U$-linear form (see 1.6.1 of [3]). Since it is an homogeneous form of degree zero, $\mathcal{K}(f, p)$ is a complex of graded $U$-modules having differentials homogeneous morphisms of degree zero. Concretely, $\mathcal{K}(f, p)=\oplus_{n \geq 0} \mathcal{K}(f, p)_{n}$ where $\mathcal{K}(f, p)_{n}$ is the following subcomplex $\left(U_{n}=0\right.$ for $\left.n<0\right)$ :

$$
\cdots \rightarrow \mathbf{\Lambda}_{2}^{A}\left(V_{p}\right) \otimes_{A} U_{n-2 p} \stackrel{\partial_{2}}{\longrightarrow} V_{p} \otimes_{A} U_{n-p} \stackrel{\partial_{1}}{\longrightarrow} U_{n} \longrightarrow 0
$$

where $\partial_{q}\left(\left(x_{1} \wedge \ldots \wedge x_{q}\right) \otimes y\right)=\sum_{i=1}^{q}(-1)^{i-1} x_{1} \wedge \ldots \wedge \widehat{x}_{i} \wedge \ldots \wedge x_{q} \otimes f_{p}\left(x_{i}\right) y$, for all $x_{i} \in V_{p}$ and $y \in U_{n-q p}$. In particular, for every $q \geq 0, H_{q}(\mathcal{K}(f, p))$ is a graded $A$-module with $H_{q}(\mathcal{K}(f, p))_{n}=H_{q}\left(\mathcal{K}(f, p)_{n}\right)$.

Theorem 2.4 Let $f: V \rightarrow U$ and $g: W \rightarrow V$ be two surjective graded morphisms of standard A-algebras. Let $\alpha: \mathbf{S}\left(U_{1}\right) \rightarrow U$ be the canonical morphism and suppose $W$ is a symmetric algebra. Given $(n \geq 2, p=1)$ or $(n \geq 2 p+1, p \geq 2)$, there are isomorphisms of A-modules

$$
H_{1}\left(\mathcal{K}(f, p)_{n}\right)=\frac{\operatorname{ker}(f \circ g)_{n}}{W_{p} \cdot \operatorname{ker}(f \circ g)_{n-p}}=\frac{\operatorname{ker} \alpha_{n}}{\mathbf{S}_{p}\left(U_{1}\right) \cdot \operatorname{ker} \alpha_{n-p}} .
$$

In particular, the module of effective n-relations of $U$ is $E(U)_{n}=H_{1}\left(\mathcal{K}(f, 1)_{n}\right)$ and the relation type of $U$ is $\operatorname{rt}(U)=\mathrm{s}\left(H_{1}(\mathcal{K}(f, 1))\right)$.

Proof. Put $h=f \circ g$. Since $n-p \geq p$, then $W_{n-p} \cdot \operatorname{ker} g_{p} \subset W_{p} \cdot \operatorname{ker} g_{n-p} \subset W_{p} \cdot \operatorname{ker} h_{n-p}$. Applying the snake lemma to the commutative diagram of exact rows 


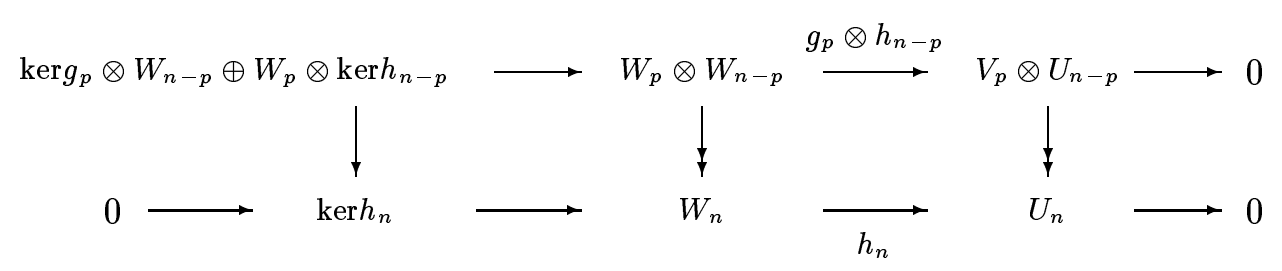

we get the exact sequence of $A$-modules

$$
0 \rightarrow\left(g_{p} \otimes h_{n-p}\right)\left(\mathcal{Z}_{1}\left(1_{W}, p\right)_{n}\right) \rightarrow \mathcal{Z}_{1}(f, p)_{n} \rightarrow \operatorname{ker} h_{n} / W_{p} \cdot \operatorname{ker} h_{n-p} \rightarrow 0
$$

where $\mathcal{Z}_{1}\left(1_{W}, p\right)_{n}, \mathcal{Z}_{1}(f, p)_{n}$ stand for the $n$-th component of the 1-cycles module of $\mathcal{K}\left(1_{W}, p\right)$, $\mathcal{K}(f, p)$. If $\mathcal{Z}_{1}\left(1_{W}, p\right)_{n}=\mathcal{B}_{1}\left(1_{W}, p\right)_{n}$ (the $n$-th component of the 1-boundaries module of $\left.\mathcal{K}\left(1_{W}, p\right)\right)$, then $\left(g_{p} \otimes h_{n-p}\right)\left(\mathcal{Z}_{1}\left(1_{W}, p\right)_{n}\right)=\mathcal{B}_{1}(f, p)_{n}$ Thus, the first isomorphism is demonstrated provided we prove $H_{1}\left(\mathcal{K}\left(1_{W}, p\right)\right)_{n}=0$ for a symmetric algebra $W$ (see next lemma). In particular, if we take $V=U$ and $f=1_{U}$, then $h=f \circ g=g$ and one of the possible choices of $h$ is the canonical morphism $\alpha$. Hence, applying twice the first equality to $\alpha$ and to any $h: W \rightarrow U$ arising from a symmetric algebra $W$, we have

$$
H_{1}\left(\mathcal{K}\left(1_{U}, p\right)_{n}\right)=\frac{\operatorname{ker} \alpha_{n}}{\mathbf{S}_{p}\left(U_{1}\right) \cdot \operatorname{ker} \alpha_{n-p}}=\frac{\operatorname{ker} h_{n}}{W_{p} \cdot \operatorname{ker} h_{n-p}} \cdot \mathbf{I}
$$

Lemma 2.5 Let $M$ be an $A$-module and $W=\mathbf{S}(M)$ the symmetric algebra of $M$. Then, for $(n \geq 1, p=1)$ or $(n \geq 2 p+1, p \geq 2), H_{1}\left(\mathcal{K}\left(1_{W}, p\right)\right)_{n}=0$.

Proof. Put $\mathbf{T}(M)$ the tensorial algebra of $M$ and $q=n-p$. Applying the snake lemma to the commutative diagram of exact rows

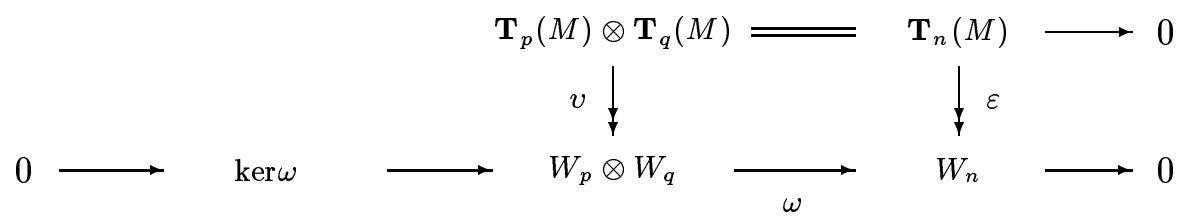

we get the exact sequence $0 \rightarrow \operatorname{ker} v \rightarrow \operatorname{ker} \varepsilon \stackrel{v}{\rightarrow} \operatorname{ker} \omega \rightarrow 0$. Thus, $\mathcal{Z}_{1}\left(1_{W}, p\right)_{n}=\operatorname{ker} \omega=$ $v(\operatorname{ker} \varepsilon)$ is the $A$-module generated by the elements

$$
\left(x_{1} \cdots x_{p-1} x_{p}\right) \otimes\left(y_{1} y_{2} \cdots y_{q}\right)-\left(x_{1} \cdots x_{p-1} y_{1}\right) \otimes\left(x_{p} y_{2} \cdots y_{q}\right),
$$

where $x_{i}, y_{j} \in M$ and $x_{1} \cdots x_{p}$ stands for the product in $W=\mathbf{S}(M)$. Clearly, if $(n \geq 1$, $p=1)$, then $\mathcal{Z}_{1}\left(1_{W}, p\right)_{n}=\mathcal{B}_{1}\left(1_{W}, p\right)_{n}$. Suppose $(n \geq 2 p+1, p \geq 2)$, i.e., $q>p$. Then, $H_{1}\left(\mathcal{K}\left(1_{W}, p\right)_{n}\right)=0$ follows from the equality:

$$
\begin{aligned}
& \left(x_{1} \cdots x_{p}\right) \otimes\left(y_{1} \cdots y_{q}\right)-\left(x_{1} \cdots x_{p-1} y_{1}\right) \otimes\left(x_{p} y_{2} \cdots y_{q}\right)= \\
& \left(x_{1} \cdots x_{p}\right) \otimes\left(y_{1} \cdots y_{q}\right)-\left(y_{2} \cdots y_{p+1}\right) \otimes\left(x_{1} \cdots x_{p} y_{1} y_{p+2} \cdots y_{q}\right)+ \\
& \left(y_{2} \cdots y_{p+1}\right) \otimes\left(x_{1} \cdots x_{p-1} y_{1} x_{p} y_{p+2} \cdots y_{q}\right)-\left(x_{1} \cdots x_{p-1} y_{1}\right) \otimes\left(x_{p} y_{2} \cdots y_{q}\right) \cdot \mathbf{I}
\end{aligned}
$$


Remark 2.6 Let $f: \mathbf{S}(F) \rightarrow \mathbf{S}(M)$ be the induced morphism on the symmetric algebras by an epimorphism $\pi: F \rightarrow M$ of $A$-modules. Then, the last three nonzero terms of $\mathcal{K}(f, p)_{p+q}, q \geq p \geq 1$, define the sequence:

$$
\mathbf{\Lambda}_{2}^{A}\left(\mathbf{S}_{p}(F)\right) \otimes_{A} \mathbf{S}_{q-p}(M) \stackrel{\partial_{2}}{\rightarrow} \mathbf{S}_{p}(F) \otimes_{A} \mathbf{S}_{q}(M) \stackrel{\partial_{1}}{\rightarrow} \mathbf{S}_{p+q}(M) \rightarrow 0,
$$

with $\partial_{2}\left(\left(x_{1} \cdots x_{p}\right) \wedge\left(y_{1} \cdots y_{p}\right) \otimes z\right)=\left(y_{1} \cdots y_{p}\right) \otimes f\left(x_{1} \cdots x_{p}\right) z-\left(x_{1} \cdots x_{p}\right) \otimes f\left(y_{1} \cdots y_{p}\right) z$ and $\partial_{1}\left(\left(x_{1} \cdots x_{p}\right) \otimes t\right)=f\left(x_{1} \cdots x_{p}\right) t, x_{i}, y_{j} \in F, z \in \mathbf{S}_{q-p}(M)$ and $t \in \mathbf{S}_{q}(M)$.

On the other hand, Micali and Roby defined (in [10]) the sequence of $A$-modules

$$
\mathbf{T}_{p+q}^{A}(F) \stackrel{\lambda}{\rightarrow} \mathbf{S}_{p}(F) \otimes_{A} \mathbf{S}_{q}(M) \stackrel{\mu}{\rightarrow} \mathbf{S}_{p+q}(M) \rightarrow 0,
$$

with $\lambda\left(x_{1} \otimes \ldots \otimes x_{p+q}\right)=\left(x_{1} \cdots x_{p}\right) \otimes f\left(x_{p+1} \cdots x_{p+q}\right)-\left(x_{1} \cdots x_{p-1} x_{p+1}\right) \otimes f\left(x_{p} x_{p+2} \cdots x_{p+q}\right)$ and $\mu=\partial_{1}$. By a similar argument to that one of the end of Lemma 2.5, one can prove that $\operatorname{Im} \partial_{2}$ is always contained in $\operatorname{Im} \lambda$ and that if $q>p$, then both modules are equal. Thus, the exactness of (2) (settled by Theorem 2.4 either for $q \geq p=1$ or either for $q>p \geq 2$ ) assures the exactness of (3). Nevertheless, if $q=p \geq 2$, then (2) might not be exact (see proof of Lemma 3.8) while (3) is always exact (see [10]).

Corollary 2.7 Let $U$ be a standard A-algebra and let $\underline{x}$ be a (possibly infinite) set of forms of degree one generating $U_{+}$. If $H_{1}(\underline{x} ; U)$ denotes the first Koszul homology group associated to $\underline{x}$, then $E(U)_{n}=H_{1}(\underline{x} ; U)_{n}$ for all $n \geq 2$. In particular, $\operatorname{rt}(U)=\mathrm{s}\left(H_{1}(\underline{x} ; U)\right)$.

Proof. Take in Theorem 2.4, $f: \mathbf{S}(F) \rightarrow U$ induced by a free presentation $F \rightarrow U_{1}$ associated to $\underline{x}$. Then, $\mathcal{K}(f, 1)=\mathcal{K}(\underline{x} ; U)$ is the usual Koszul complex associated to the elements $\underline{x}$.

Remark 2.8 Using duality between Koszul homology and cohomology (see 1.6.10 of [3]) we recover Schenzel's result $\mathrm{rt}(U)=\mathrm{s}\left(H^{d-1}(\underline{x} ; U)\right)+d$, when $\underline{x}$ is finite of cardinal $d$ (see $[13])$.

Remark 2.9 Let $I$ be an ideal of $A$ and $\mathcal{R}(I)=\oplus_{n \geq 0} I^{n} t^{n}$ its Rees algebra. Take $f=1_{\mathcal{R}}$, the identity on $\mathcal{R}(I)$, in Theorem 2.4. Then,

$$
\mathcal{Z}_{1}(f, p)_{n}=\operatorname{ker}\left(I^{p} \otimes I^{n-p} \rightarrow I^{n}\right)=\operatorname{Tor}_{1}^{A}\left(A / I^{p}, I^{n-p}\right),
$$

which is known to be isomorphic to $Z_{1} \cap I^{n-p} F / I^{n-p} Z_{1}$, where $0 \rightarrow Z_{1} \rightarrow F \rightarrow I^{p} \rightarrow 0$ is a presentation of $I^{p}$ with $F$ free (see, e.g., 2.5 of [8]). Moreover, via the same isomorphism

$$
\mathcal{B}_{1}(f, p)_{n}=\operatorname{Im}\left(\Lambda_{2}^{A}\left(I^{p}\right) \otimes I^{n-2 p} \rightarrow I^{p} \otimes I^{n-p}\right)=I^{n-2 p} B_{1} / I^{n-p} Z_{1} .
$$

Thus, by Theorem 2.4, we have

$$
H_{1}(f, p)_{n}=\frac{\operatorname{ker} \alpha_{n}}{\mathbf{S}_{p}(I) \cdot \operatorname{ker} \alpha_{n-p}}=\frac{Z_{1} \cap I^{n-p} F}{I^{n-2 p} B_{1}},
$$

which reproves an earlier result of Kühl (see 1.2 of [9]). 


\section{Some applications}

The purpose of this section is to give some applications of Lemma 2.1 and Theorem 2.4.

Example 3.1 Cyclic StANDARD algebras Let $U$ be a cyclic standard $A$-algebra generated by a degree one form $x \in U_{1}$. Put $f: A[t] \rightarrow U$ with $f(t)=x$ in Theorem 2.4. Then, $E(U)_{n}=H_{1}\left(\mathcal{K}(f, 1)_{n}\right)=(0: x) \cap U_{n-1}$ and $\operatorname{rt}(U)=\min \left\{r \geq 1 \mid\left(0: x^{r+1}\right)=\left(0: x^{r}\right)\right\}$.

Example 3.2 Change of BASE RING Let $U$ be a standard $A$-algebra and let $\varphi: A \rightarrow$ $B$ be a homomorphism of rings. Take $f: V \rightarrow U$ any surjective graded morphism of standard $A$-algebras in Theorem 2.4. It induces $f \otimes 1: V \otimes_{A} B \rightarrow U \otimes_{A} B$. Since $\mathcal{K}(f \otimes 1, p)_{n}=\mathcal{K}(f, p)_{n} \otimes_{A} B$, one can deduce $\operatorname{rt}\left(U \otimes_{A} B\right) \leq \operatorname{rt}(U)$. If $\varphi$ is flat, then $H_{1}\left(\mathcal{K}(f \otimes 1, p)_{n}\right)=H_{1}\left(\mathcal{K}(f, p)_{n}\right) \otimes_{A} B$. In particular, $\operatorname{rt}(U)=\sup \left\{\operatorname{rt}\left(U_{\mathfrak{p}}\right) \mid \mathfrak{p} \in \operatorname{Spec}(A)\right\}$. If $\varphi$ is faithfully flat, then $\operatorname{rt}\left(U \otimes_{A} B\right)=\operatorname{rt}(U)$. In particular, via the Nagata morphism $A \rightarrow A[t]_{\mathfrak{m}[t]}=B, \mathfrak{m}$ a maximal ideal of $A$, one can always suppose, when calculating the relation type of $U$, that $A$ is a local ring of maximal $\mathfrak{m}$ and residual field $A / \mathfrak{m}=k$ infinite.

Let $I$ be an ideal of $A$ and $\mathcal{G}(I)=\oplus_{n \geq 0} I^{n} / I^{n+1}$ its associated graded ring. Since $\mathcal{G}(I)=\mathcal{R}(I) \otimes_{A} A / I$, then (by 3.2) $\operatorname{rt}(\mathcal{G}(I)) \leq \operatorname{rt}(\mathcal{R}(I))=\operatorname{rt}(I)$. In [14], Valla showed that if $\operatorname{rt}(\mathcal{G}(I))=1$, then $\operatorname{rt}(I)=1$ too. Next proposition is a generalization of that result.

Proposition 3.3 If $I$ is an ideal, there exists $E(I)_{n+1} \rightarrow E(I)_{n} \rightarrow E(\mathcal{G}(I))_{n} \rightarrow 0$, exact sequence of $A$-modules, for all $n \geq 2$. In particular, if $\operatorname{rt}(I)<\infty$, then $\operatorname{rt}(\mathcal{G}(I))=\operatorname{rt}(I)$.

Proof. If $1_{\mathcal{R}}, 1_{\mathcal{G}}$, denote the identity on $\mathcal{R}(I), \mathcal{G}(I)$, respectively, then for each $n \geq 1$, there is an exact sequence of complexes $\mathcal{K}\left(1_{\mathcal{R}}, 1\right)_{n+1} \rightarrow \mathcal{K}\left(1_{\mathcal{R}}, 1\right)_{n} \rightarrow \mathcal{K}\left(1_{\mathcal{G}}, 1\right)_{n} \rightarrow 0$. Since the 0 -th component of the first morphism is injective and $H_{0}\left(\mathcal{K}\left(1_{\mathcal{R}}, 1\right)_{n+1}\right)=0$, we have enough to deduce the exact sequence $E(I)_{n+1} \rightarrow E(I)_{n} \rightarrow E(\mathcal{G}(I))_{n} \rightarrow 0$. In particular, if $\operatorname{rt}(I)<\infty$, one can proceed by decreasing induction.

Remark 3.4 If $\operatorname{rt}(I)=\infty$, then 3.3 might be false as Example 4.4 of [11] shows. Note that, as a consequence of next proposition, we will see that for the irrelevant ideal of a standard algebra hypothesis $\operatorname{rt}(I)<\infty$ can be removed.

Proposition 3.5 Let $U$ be a standard A-algebra and let $U_{+}=\oplus_{n>0} U_{n}$ denote its irrelevant ideal. Take $f: W \rightarrow U$ a surjective graded morphism of standard A-algebras with $W$ a symmetric algebra. Given $(n \geq 2, p=1)$ or $(n \geq 3, p \geq 2)$, the module of effective $n$-relations of $U_{+}^{p}$ is

$$
E\left(U_{+}^{p}\right)_{n}=\bigoplus_{q \geq n p} \frac{\operatorname{ker} f_{q}}{W_{p} \cdot \operatorname{ker} f_{q-p}} .
$$

In particular, $E\left(U_{+}^{p}\right)_{n}=0$ if, and only if, $\operatorname{rt}\left(U_{+}^{p}\right) \leq n-1$. For $p=1, \operatorname{rt}(U)=\operatorname{rt}\left(U_{+}\right)$. Moreover, $U_{+}$is a syzygetic ideal if, and only if, $U$ is a symmetric algebra. 
Proof. Let $g: \mathbf{S}^{U}\left(U_{p} \otimes_{A} U\right) \rightarrow \mathcal{R}\left(U_{+}^{p}\right)$ be induced by the natural epimorphism of $A$ modules $U_{p} \otimes_{A} U \rightarrow U_{+}^{p}$. It is not hard to see $\mathcal{K}(g, 1)_{n}=\oplus_{i \geq 0} \mathcal{K}\left(1_{U}, p\right)_{n p+i}$. Moreover, if $(n \geq 2, p=1)$, then $n p+i \geq 2$ and if $(n \geq 3, p \geq 2)$, then $n p+i \geq 2 p+1$. Therefore, by Theorem 2.4,

$$
E\left(U_{+}^{p}\right)_{n}=H_{1}\left(\mathcal{K}(g, 1)_{n}\right)=\bigoplus_{i \geq 0} H_{1}\left(\mathcal{K}\left(1_{U}, p\right)_{n p+i}\right)=\bigoplus_{i \geq 0} \frac{\operatorname{ker} f_{n p+i}}{W_{p} \cdot \operatorname{ker} f_{(n-1) p+i}}=\bigoplus_{q \geq n p} \frac{\operatorname{ker} f_{q}}{W_{p} \cdot \operatorname{ker} f_{q-p}} .
$$

In particular, $E\left(U_{+}^{p}\right)_{n} \supset E\left(U_{+}^{p}\right)_{n+1}$. Thus, $E\left(U_{+}^{p}\right)_{n}=0$ is equivalent to $\operatorname{rt}\left(U_{+}^{p}\right) \leq n-1$. For $p=1$ and $n \geq 2, E\left(U_{+}\right)_{n}=\oplus_{i \geq 0} \operatorname{ker} f_{n+i} / W_{1} \cdot \operatorname{ker} f_{n-1+i}=\oplus_{i \geq 0} E(U)_{n+i}=\oplus_{q \geq n} E(U)_{q}$. In particular, $\operatorname{rt}(U)=\mathrm{s}(E(U))=\mathrm{s}\left(E\left(U_{+}\right)\right)=\operatorname{rt}\left(U_{+}\right)$. Moreover, $E\left(U_{+}\right)_{2}=\oplus_{q \geq 2} E(U)_{q}=$ $E(U)$. Thus, $U_{+}$be syzygetic is equivalent to $U$ be a symmetric algebra.

Now, let us focus our attention into the relation type of Veronese subrings. Let $U$ be a standard $A$-algebra. Recall that the $p$-th Veronese subring of $U$ is defined to be the standard $A$-algebra $U^{(p)}=\oplus_{n \geq 0} U_{n p}$. Clearly, if $f: V \rightarrow U$ is a (surjective) graded morphism of standard $A$-algebras, then it induces $f^{(p)}: V^{(p)} \rightarrow U^{(p)}$ another (surjective) graded morphism of standard $A$-algebras.

Lemma 3.6 Let $f: V \rightarrow U$ be a surjective graded morphism of standard A-algebras. Then, for all $p \geq 1, \mathrm{~s}\left(E\left(f^{(p)}\right)\right) \leq 1+[(\mathrm{s}(E(f))-1) / p]$ ([a] is the integer part of a).

Proof. Write $\mathrm{s}(E(f))-1=p a+b$ with $0 \leq b<p$. So $[(\mathrm{s}(E(f))-1) / p]=a$. Take $n \geq 2+a$. Then $(n-1) p \geq p a+p \geq \mathrm{s}(E(f))$. Thus, $\operatorname{ker} f_{n p}=V_{1} \cdot \operatorname{ker} f_{n p-1}=\ldots=V_{p} \cdot \operatorname{ker} f_{(n-1) p}$ and hence $\mathrm{s}\left(E\left(f^{(p)}\right)\right) \leq 1+a$.

Lemma 3.7 Let $U$ be a standard A-algebra and let $f: V \rightarrow U$ be a symmetric presentation of $U$. If $(n \geq 2, p=1)$ or $(n \geq 3, p \geq 2)$, then the module of effective $n$-relations of $U^{(p)}$ is

$$
E\left(U^{(p)}\right)_{n}=\frac{\operatorname{ker} f_{n p}}{V_{p} \cdot \operatorname{ker} f_{(n-1) p}} .
$$

Proof. Take $g: \mathbf{S}\left(V_{p}\right) \rightarrow U^{(p)}$ induced by $f_{p}: V_{p} \rightarrow U_{p}$ in degree one. We have $\mathcal{K}(g, 1)_{n}=$ $\mathcal{K}(f, p)_{n p}$. Moreover, if $(n \geq 2, p=1)$, then $n p \geq 2$, and if $(n \geq 3, p \geq 2)$, then $n p \geq$ $2 p+1$. Thus, by Theorem 2.4, $E\left(U^{(p)}\right)_{n}=H_{1}\left(\mathcal{K}(g, 1)_{n}\right)=H_{1}\left(\mathcal{K}(f, p)_{n p}\right)=\left(\operatorname{ker} f_{n p}\right) /\left(V_{p}\right.$. $\left.\operatorname{ker} f_{(n-1) p}\right)$.

Lemma 3.8 Let $M$ be an A-module and $\mathbf{S}(M)$ its symmetric algebra. Then, for all $p \geq 1$, $\operatorname{rt}\left(\mathbf{S}(M)^{(p)}\right) \leq 2$. Moreover, if $p \geq 2$ and $M$ is finitely generated, then $\operatorname{rt}\left(\mathbf{S}(M)^{(p)}\right)=1$ if, and only if, $M$ is locally cyclic.

Proof. By Lemma 3.7, $E\left(\mathbf{S}(M)^{(p)}\right)_{n}=0$ for all $n \geq 3$. Thus, $\operatorname{rt}\left(\mathbf{S}(M)^{(p)}\right) \leq 2$. Suppose $p \geq 2$ and $(A, \mathfrak{m}, k)$ is local (see 3.2). If $M$ is cyclic, then $\mathbf{S}(M)^{(p)}=\mathbf{S}\left(\mathbf{S}_{p}(M)\right.$ ) and $\operatorname{rt}\left(\mathbf{S}(M)^{(p)}\right)=1$. Conversely, suppose $M$ finitely generated, but not cyclic. Take $x, y$ part of a basis of $M \otimes k$ and $x^{p}, y^{p}, x^{p-1} y$ in $\mathbf{S}_{p}(M) \otimes k$. Then, $z=x^{p} \otimes y^{p}-x^{p-1} y \otimes$ 
$x y^{p-1} \in \mathcal{Z}_{1}\left(f \otimes 1_{k}, 1\right)_{2}$. Moreover, looking at the components of an element in a $k$-basis of $\mathcal{B}_{1}\left(f \otimes 1_{k}, 1\right)_{2}$, one sees that $z \notin \mathcal{B}_{1}\left(f \otimes 1_{k}, 1\right)_{2}$. Thus, $H_{1}\left(\mathcal{K}\left(f \otimes 1_{k}, 1\right)_{2}\right) \neq 0$, hence (by 3.2) $H_{1}\left(\mathcal{K}(f, 1)_{2}\right) \neq 0$ and $\operatorname{rt}\left(\mathbf{S}(M)^{(p)}\right)=2$.

Remark 3.9 Let $I$ be an ideal of linear type finitely generated, but not locally principal. Then, by Lemma $3.8, \operatorname{rt}\left(I^{p}\right)=2$ for all $p \geq 2$, which reproves 2.6 of [7].

Theorem 3.10 Let $U$ be a standard A-algebra. Then, $\operatorname{rt}\left(U^{(p)}\right) \leq \max (1+[(\operatorname{rt}(U)-1) / p], 2)$ for all $p \geq 1$. Moroever, if $U$ is finitely generated and $p \geq 2$, then $\operatorname{rt}\left(U^{(p)}\right)=1$ if, and only if, $U_{p}$ is locally generated by a d-sequence of length 1.

Proof. Let $\alpha: \mathbf{S}\left(U_{1}\right) \rightarrow U$ be the canonical morphism. Put $g: \mathbf{S}\left(\mathbf{S}_{p}\left(U_{1}\right)\right) \rightarrow \mathbf{S}\left(U_{1}\right)^{(p)}$ and $f=\alpha^{(p)}$. Then, by Lemma 2.1, $\operatorname{rt}\left(U^{(p)}\right) \leq \max (\mathrm{s}(E(f)), \mathrm{s}(E(g)))$ and, by Lemmas 3.6 and 3.8, we prove the inequality. Suppose $p \geq 2$ and $U$ finitely generated. By 3.2, one can suppose that $(A, \mathfrak{m}, k)$ is a local ring of infinite residual field $k$. If $U_{p}$ is generated by a $d$-sequence of length 1 , then (by 3.1) $\operatorname{rt}\left(U^{(p)}\right)=1$. Conversely, suppose $\operatorname{rt}\left(U^{(p)}\right)=1$. Take $V=U \otimes k$, so $V^{(p)}=U^{(p)} \otimes k$ and $\operatorname{rt}\left(V^{(p)}\right) \leq \operatorname{rt}\left(U^{(p)}\right)=1$. Therefore, $V^{(p)}$ is a polynomial ring of Krull dimension $l=\mu\left(V_{p}\right)=\operatorname{dim} V^{(p)}=\operatorname{dim} V$ (since $V^{(p)} \subset V$ is an integral extension). Take $W \subset V$ a graded Noether normalization (it exists since $k$ is infinite, see 1.5.17 of [3]). Thus, $\operatorname{dim} W=\operatorname{dim} V=l$ and so $\left(\begin{array}{c}l+p-1 \\ p\end{array}\right)=\mu\left(W_{p}\right) \leq \mu\left(V_{p}\right)=l$, which forces $l=1$. Hence, $\mu\left(U_{p}\right)=\mu\left(V_{p}\right)=1, U_{p}=A x$ is cyclic and, by 3.1 again, $x$ is a $d$-sequence.

Remark 3.11 The inequality of 3.10 was firstly proved by Backelin and Fröberg for finitely generated $k$-algebras (see [2]). Recently, Johnston and Katz showed a very similar statement to that of 3.10, but for $U=\mathcal{R}(I)$ the Rees algebra of an ideal $I$ (see [7]). Since $\mathcal{G}(I)^{(p)}=$ $\mathcal{R}(I)^{(p)} \otimes A / I=\mathcal{R}\left(I^{p}\right) \otimes A / I$, then (by 3.2) $\operatorname{rt}\left(\mathcal{G}(I)^{(p)}\right) \leq \operatorname{rt}\left(I^{p}\right)$. In particular, for $I=U_{+}$ the irrelevant ideal of a standard algebra $U, \mathcal{G}(I)=U$ and $\operatorname{rt}\left(U^{(p)}\right) \leq \operatorname{rt}\left(U_{+}^{p}\right)$. Thus, Johnston-Katz's result implies Backelin-Fröberg's result and the inequality of 3.10, when $U$ is a Noetherian ring. Nevertheless, the whole Theorem 3.10 can not be deduced directly from earlier results since, in general, $\operatorname{rt}\left(U^{(p)}\right) \neq \operatorname{rt}\left(U_{+}^{p}\right)$ as next example shows.

Example 3.12 Put $U=k[x, y, z] / J$ with $J=\left(x^{3} y, x y^{3}, z^{4}, x^{2} y^{2} z^{3}\right)$. Then, $\operatorname{rt}(U)=7$, $\operatorname{rt}\left(U^{(2)}\right)=2$ and $\operatorname{rt}\left(U_{+}^{2}\right)=3$ (remark that $\left.\max (1+[(\operatorname{rt}(U)-1) / 2], 2)=4\right)$. Indeed, since $E(U)_{n}=\operatorname{ker} \alpha_{n} / U_{1} \cdot \operatorname{ker} \alpha_{n-1}, \alpha: \mathbf{S}\left(U_{1}\right) \rightarrow U$ the canonical morphism, then $E(U)_{n}=0$ for all $n \geq 2, n \neq 4,7$ and $E(U)_{4}=k^{\oplus 3}$ and $E(U)_{7}=k$. Thus, $\operatorname{rt}(U)=\mathrm{s}(E(U))=7$. Since $\operatorname{ker} \alpha_{8} \subset F_{4}(\operatorname{ker} \alpha)$, then, by Lemma 3.7, $E\left(U^{(2)}\right)_{n}=\operatorname{ker} \alpha_{2 n} / \mathbf{S}_{2}\left(U_{1}\right) \cdot \operatorname{ker} \alpha_{2(n-1)}=0$ for all $n \geq 3$. Thus, $\operatorname{rt}\left(U^{(2)}\right) \leq 2$. Moreover, $\operatorname{rt}\left(U^{(2)}\right)=2$ since $U_{2}$ is not locally cyclic (see Theorem 3.10). Besides, using Proposition 3.5, $E\left(U_{+}^{2}\right)_{4}=\oplus_{q \geq 8}\left(\operatorname{ker} \alpha_{q} / \mathbf{S}_{2}\left(U_{1}\right) \operatorname{ker} \alpha_{q-2}\right)=0$, so $\operatorname{rt}\left(U_{+}^{2}\right) \leq 3$. But, since $\operatorname{ker} \alpha_{7} \neq \mathbf{S}_{2}\left(U_{1}\right) \cdot \operatorname{ker} \alpha_{5}, E\left(U_{+}^{2}\right)_{3}=\oplus_{q \geq 6}\left(\operatorname{ker} \alpha_{q} / \mathbf{S}_{2}\left(U_{1}\right) \operatorname{ker} \alpha_{q-2}\right) \neq$ 0 . Hence, $\operatorname{rt}\left(U_{+}^{2}\right)=3$. 


\section{Conditions on the generators}

In this section we characterize, in terms of a system of generators, which ideals have module of effective $n$-relations zero. Our work here is inspired in previous results by Costa, see [5] and [6]. Concretely, in [6], it was defined a sequence of linear type as a sequence of elements $x_{1}, \ldots, x_{d}$ such that the ideals $\left(x_{1}, \ldots, x_{i}\right)$ are of linear type for $i=1, \ldots, d$. As a consequence of the main result of this section (see 4.7), we get a new characterization of sequences of linear type involving annihilator ideals (see 4.9). For an ideal $I$ generated by $d$ elements $x_{1}, \ldots, x_{d}$, we will denote by $I_{i_{1}, \ldots, i_{s}}$ the ideal generated by the $x_{j}$, where $j \notin\left\{i_{1}, \ldots, i_{s}\right\}$. For an $A$-module $M$, we will denote by $\mathcal{A}_{d}(M)$ the set of alternating $d \times d$ matrices with coefficients in $M$.

Lemma 4.1 Let $I$ be generated by d elements $x_{1}, \ldots, x_{d}$ and take $n \geq 2$. Then, $E(I)_{n}=0$ if, and only if, for all $\left(a_{1}, \ldots, a_{d}\right) \in\left(I^{n-1}\right)^{\oplus d}$ with $a_{1} x_{1}+\ldots+a_{d} x_{d}=0$, there exists $\left(b_{i, j}\right) \in \mathcal{A}_{d}\left(I^{n-2}\right)$ such that

$$
\left(\begin{array}{c}
a_{1} \\
a_{2} \\
\vdots \\
a_{d}
\end{array}\right)=\left(\begin{array}{cccc}
0 & b_{1,2} & \ldots & b_{1, d} \\
-b_{1,2} & 0 & \ldots & b_{2, d} \\
\vdots & \vdots & \ddots & \vdots \\
-b_{1, d} & -b_{2, d} & \ldots & 0
\end{array}\right)\left(\begin{array}{c}
x_{1} \\
x_{2} \\
\vdots \\
x_{d}
\end{array}\right)
$$

Proof. By Corollary 2.7, $E(I)_{n}=H_{1}(\underline{x t} ; \mathcal{R}(I))_{n}$, where $\mathcal{K}(\underline{x t} ; \mathcal{R}(I))_{n}$ is the $n$-th component of the Koszul complex associated to the elements $x_{1} t, \ldots, x_{d} t$ in $\mathcal{R}(I)=\oplus_{n \geq 0} I^{n} t^{n}$. That

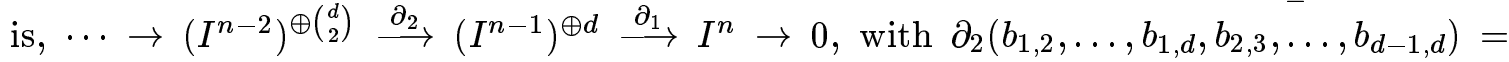
$\left(a_{1}, \ldots, a_{d}\right)$ defined by $\left(\begin{array}{c}a_{1} \\ a_{2} \\ \vdots \\ a_{d}\end{array}\right)=\left(\begin{array}{cccc}0 & b_{1,2} & \ldots & b_{1, d} \\ -b_{1,2} & 0 & \ldots & b_{2, d} \\ \vdots & \vdots & \ddots & \vdots \\ -b_{1, d} & -b_{2, d} & \ldots & 0\end{array}\right)\left(\begin{array}{c}x_{1} \\ x_{2} \\ \vdots \\ x_{d}\end{array}\right)$, and $\partial_{1}\left(a_{1}, \ldots, a_{d}\right)=$ $a_{1} x_{1}+\cdots+a_{d} x_{d}$.

Lemma 4.2 Let $I$ be generated by d elements $x_{1}, \ldots, x_{d}$ and take $n \geq 2$. If $E(I)_{n}=0$, then $I_{1} I^{n-1}: x_{1}^{n}=I_{1} I^{n-2}: x_{1}^{n-1}$.

Proof. If $a \in I_{1} I^{n-1}: x_{1}^{n}$, then $a x_{1}^{n}=a_{2} x_{2}+\cdots+a_{d} x_{d}, a_{i} \in I^{n-1}$. In particular, (by 4.1) $\left(\begin{array}{c}a x_{1}^{n-1} \\ -a_{2} \\ \vdots \\ -a_{d}\end{array}\right)=\left(\begin{array}{cccc}0 & b_{1,2} & \ldots & b_{1, d} \\ -b_{1,2} & 0 & \ldots & b_{2, d} \\ \vdots & \vdots & \ddots & \vdots \\ -b_{1, d} & -b_{2, d} & \ldots & 0\end{array}\right)\left(\begin{array}{c}x_{1} \\ x_{2} \\ \vdots \\ x_{d}\end{array}\right), b_{i, j} \in I^{n-2}$. Thus $a x_{1}^{n-1} \in I_{1} I^{n-2} \cdot \mathbf{I}$

Remark 4.3 If $d=1$, then the necessary condition of Lemma 4.2 becomes $0: x_{1}^{n}=0$ : $x_{1}^{n-1}$, which is known to be sufficient to assure $E(I)_{n}=0$ (see Example 3.1).

Lemma 4.4 Let I be generated by d elements $x_{1}, \ldots, x_{d}(d \geq 2)$ and $n \geq 2$. If $E(I)_{n}=0$, then

$$
\left(0: x_{1}\right) \cap I^{n-1}=\left\{\sum_{i=2}^{d} a_{i} x_{i} \mid a_{i} \in I^{n-2}, x_{1}\left(\begin{array}{c}
a_{2} \\
\vdots \\
a_{d}
\end{array}\right)=\left(b_{i, j}\right)\left(\begin{array}{c}
x_{2} \\
\vdots \\
x_{d}
\end{array}\right) \text { for }\left(b_{i, j}\right) \in \mathcal{A}_{d-1}\left(I_{1}^{n-2}\right)\right\} .
$$


In particular, if $d=2$ and $E(I)_{n}=0$, then $\left(0: x_{1}\right) \cap I^{n-1}=x_{2}\left(\left(0: x_{1}\right) \cap I^{n-2}\right)$ and $\left(0: x_{1} x_{2}\right) \cap I^{n-2}=\left(0: x_{1}\right) \cap I^{n-2}+\left(0: x_{2}\right) \cap I^{n-2}$.

Proof. If $a \in\left(0: x_{1}\right) \cap I^{n-1}$, then (by 4.1) $\left(\begin{array}{c}a \\ 0 \\ \vdots \\ 0\end{array}\right)=\left(\begin{array}{cccc}0 & b_{1,2} & \ldots & b_{1, d} \\ -b_{1,2} & 0 & \ldots & b_{2, d} \\ \vdots & \vdots & \ddots & \vdots \\ -b_{1, d} & -b_{2, d} & \ldots & 0\end{array}\right)\left(\begin{array}{c}x_{1} \\ x_{2} \\ \vdots \\ x_{d}\end{array}\right)$ for some $\left(b_{i, j}\right) \in \mathcal{A}_{d}\left(I^{n-2}\right)$. Thus, $a=b_{1,2} x_{2}+\cdots+b_{1, d} x_{d}$ and

$$
\begin{gathered}
x_{1}\left(\begin{array}{c}
b_{1,2} \\
b_{1,3} \\
\vdots \\
b_{1, d}
\end{array}\right)=\left(\begin{array}{cccc}
0 & b_{2,3} & \ldots & b_{2, d} \\
-b_{2,3} & 0 & \ldots & b_{3, d} \\
\vdots & \vdots & \ddots & \vdots \\
-b_{2, d} & -b_{3, d} & \ldots & 0
\end{array}\right)= \\
=\left(\begin{array}{cccc}
0 & c_{2,3} & \ldots & c_{2, d} \\
-c_{2,3} & 0 & \ldots & c_{3, d} \\
\vdots & \vdots & \ddots & \vdots \\
-c_{2, d} & -c_{3, d} & \ldots & 0
\end{array}\right)\left(\begin{array}{c}
x_{2} \\
x_{3} \\
\vdots \\
x_{d}
\end{array}\right)+x_{1}\left(\begin{array}{cccc}
0 & e_{2,3} & \ldots & e_{2, d} \\
-e_{2,3} & 0 & \ldots & e_{3, d} \\
\vdots & \vdots & \ddots & \vdots \\
-e_{2, d} & -e_{3, d} & \ldots & 0
\end{array}\right)\left(\begin{array}{c}
x_{2} \\
x_{3} \\
\vdots \\
x_{d}
\end{array}\right)
\end{gathered}
$$

with $c_{i, j} \in I_{1}^{n-2}, e_{i, j} \in I^{n-3}$ and $b_{i, j}=c_{i, j}+x_{1} e_{i, j}$ (if $\left.n=2, I^{n-3}=0\right)$. Put

$$
\left(\begin{array}{c}
a_{2} \\
a_{3} \\
\vdots \\
a_{d}
\end{array}\right)=\left(\begin{array}{c}
b_{1,2} \\
b_{1,3} \\
\vdots \\
b_{1, d}
\end{array}\right)-\left(\begin{array}{cccc}
0 & e_{2,3} & \ldots & e_{2, d} \\
-e_{2,3} & 0 & \ldots & e_{3, d} \\
\vdots & \vdots & \ddots & \vdots \\
-e_{2, d} & -e_{3, d} & \ldots & 0
\end{array}\right)\left(\begin{array}{c}
x_{2} \\
x_{3} \\
\vdots \\
x_{d}
\end{array}\right)
$$

Then $x_{1}\left(\begin{array}{c}a_{2} \\ a_{3} \\ \vdots \\ a_{d}\end{array}\right)=\left(\begin{array}{cccc}0 & c_{2,3} & \ldots & c_{2, d} \\ -c_{2,3} & 0 & \ldots & c_{3, d} \\ \vdots & \vdots & \ddots & \vdots \\ -c_{2, d} & -c_{3, d} & \ldots & 0\end{array}\right)\left(\begin{array}{c}x_{2} \\ x_{3} \\ \vdots \\ x_{d}\end{array}\right)$ and $a=a_{2} x_{2}+\cdots+a_{d} x_{d}$. Conversely, if $a=a_{2} x_{2}+\cdots+a_{d} x_{d}, a_{i} \in I^{n-2}$, with $x_{1}\left(\begin{array}{c}a_{2} \\ a_{3} \\ \vdots \\ a_{d}\end{array}\right)=\left(\begin{array}{cccc}0 & b_{2,3} & \ldots & b_{2, d} \\ -b_{2,3} & 0 & \ldots & b_{3, d} \\ \vdots & \vdots & \ddots & \vdots \\ -b_{2, d} & -b_{3, d} & \ldots & 0\end{array}\right)\left(\begin{array}{c}x_{2} \\ x_{3} \\ \vdots \\ x_{d}\end{array}\right)$, $b_{i, j} \in I_{1}^{n-2}$, then clearly $a x_{1}=0$. In particular, for $d=2$, we have that $\left(0: x_{1}\right) \cap I^{n-1}=$ $x_{2}\left(\left(0: x_{1}\right) \cap I^{n-2}\right)$. Moreover, if $a \in\left(0: x_{1} x_{2}\right) \cap I^{n-2}$, then $a x_{2} \in\left(0: x_{1}\right) \cap I^{n-1}$ and hence $a x_{2}=x_{2} b$ for some $b \in\left(0: x_{1}\right) \cap I^{n-2}$. Thus, $a=b+(a-b)$ where $b \in\left(0: x_{1}\right) \cap I^{n-2}$ and $(a-b) \in\left(0: x_{2}\right) \cap I^{n-2}$.

Proposition 4.5 Let $I$ be generated by $x_{1}, x_{2}$ and $n \geq 2$. Then, $E(I)_{n}=0$ if, and only if,

(i) $x_{2} I^{n-1}: x_{1}^{n}=x_{2} I^{n-2}: x_{1}^{n-1}$,

(ii) $\left(0: x_{2}\right) \cap I^{n-1}=x_{1}\left(\left(0: x_{2}\right) \cap I^{n-2}\right)$.

Proof. By Lemmas 4.2 and 4.4, $E(I)_{n}=0$ implies conditions $(i)$ and (ii). Conversely, suppose $(i)$ and $(i i)$ are fulfilled and let us prove $E(I)_{n}=0$ via Lemma 4.1. Take $\left(a_{1}, a_{2}\right) \in$ 
$\left(I^{n-1}\right)^{\oplus 2}$ with $a_{1} x_{1}+a_{2} x_{2}=0$. Since $I^{n-1}=A x_{1}^{n-1}+x_{2} I^{n-2}, a_{1}=b_{1} x_{1}^{n-1}+b_{2} x_{2}$ with $b_{1} \in A$ and $b_{2} \in I^{n-2}$. Then, $0=a_{1} x_{1}+a_{2} x_{2}=b_{1} x_{1}^{n}+\left(a_{2}+b_{2} x_{1}\right) x_{2}$ and $b_{1} \in x_{2} I^{n-1}$ : $x_{1}^{n}=x_{2} I^{n-2}: x_{1}^{n-1}$ (by $\left.(i)\right)$. So $b_{1} x_{1}^{n-1}=c_{2} x_{2}, c_{2} \in I^{n-2}$, and $a_{1}=\left(b_{2}+c_{2}\right) x_{2}$. Therefore, $0=a_{1} x_{1}+a_{2} x_{2}=\left(a_{2}+b_{2} x_{1}+c_{2} x_{1}\right) x_{2}$. So, by $(i i),\left(a_{2}+b_{2} x_{1}+c_{2} x_{1}\right) \in\left(0: x_{2}\right) \cap I^{n-1}=$ $x_{1}\left(\left(0: x_{2}\right) \cap I^{n-2}\right)$. We thus have $a_{2}+b_{2} x_{1}+c_{2} x_{1}=c_{1} x_{1}$ with $c_{1} \in I^{n-2}$ and $c_{1} x_{2}=0$. That is, $a_{2}=\left(c_{1}-b_{2}-c_{2}\right) x_{1}$ and $a_{1}=\left(b_{2}+c_{2}\right) x_{2}=\left(b_{2}+c_{2}-c_{1}\right) x_{2}$.

Remark 4.6 Proposition 4.5 generalizes Theorem 2 of [5] and his later improvement in Theorem 4 of [6]. Remark that for an ideal $I=\left(x_{1}, x_{2}\right)$, be of linear type does not imply $I_{1}=\left(x_{2}\right)$ or $I_{2}=\left(x_{1}\right)$ be of linear type (see Example 3.3 of [12] where $I=\left(x_{1}, x_{2}\right)$ of linear type is constructed with $0: x^{2} \neq 0: x$ for all $x \in I$ ).

Theorem 4.7 Let $I$ be generated by $d$ elements $x_{1}, \ldots, x_{d}(d \geq 3)$ and take $n \geq 2$. Then $E(I)_{n}=0$ if, and only if,

(i) $I_{i} I^{n-1}: x_{i}^{n}=I_{i} I^{n-2}: x_{i}^{n-1}$ for all $i=1, \ldots, d$,

(ii) $\left(\left(\sum_{1 \leq i<j \leq d-1} x_{i} x_{j} I_{d}^{n-2}\right): x_{d}\right) \cap I^{n-1}=\sum_{i=1}^{d-1} x_{i}\left(\left(I_{i, d} I_{d}^{n-2}: x_{d}\right) \cap I^{n-2}\right)$,

(iii) If $\left(\begin{array}{c}a_{1} \\ \vdots \\ a_{d-1}\end{array}\right)=\left(\begin{array}{ccc}0 & \ldots & b_{1, d-1} \\ \vdots & \ddots & \vdots \\ b_{d-1,1} & \ldots & 0\end{array}\right)\left(\begin{array}{c}x_{1} \\ \vdots \\ x_{d-1}\end{array}\right)$ with $\sum_{i=1}^{d-1} a_{i} x_{i}=0$ and $b_{i, j} \in I_{d}^{n-2}$, then

$$
\left(\begin{array}{c}
a_{1} \\
\vdots \\
a_{d-1} \\
0
\end{array}\right)=\left(\begin{array}{cccc}
0 & \ldots & c_{1, d-1} & c_{1, d} \\
\vdots & \ddots & \vdots & \vdots \\
-c_{1, d-1} & \ldots & 0 & c_{d-1, d} \\
-c_{1, d} & \ldots & -c_{d-1, d} & 0
\end{array}\right)\left(\begin{array}{c}
x_{1} \\
\vdots \\
x_{d-1} \\
x_{d}
\end{array}\right) \text { for some }\left(c_{i, j}\right) \in \mathcal{A}_{d}\left(I^{n-2}\right) \text {. }
$$

Proof. By Lemmas 4.2 and 4.1, $E(I)_{n}=0$ clearly implies conditions $(i)$ and $(i i i)$. Let us prove $(i i)$ provided $E(I)_{n}=0$. Take $a \in \sum_{i=1}^{d-1} x_{i}\left(\left(I_{i, d} I_{d}^{n-2}: x_{d}\right) \cap I^{n-2}\right)$, so $a=$ $a_{1} x_{1}+\cdots+a_{d-1} x_{d-1}$ with $a_{i} \in I^{n-2}$ and

$$
x_{d}\left(\begin{array}{c}
a_{1} \\
a_{2} \\
\vdots \\
a_{d-1}
\end{array}\right)=\left(\begin{array}{cccc}
0 & b_{1,2} & \ldots & b_{1, d-1} \\
b_{2,1} & 0 & \ldots & b_{2, d-1} \\
\vdots & \vdots & \ddots & \vdots \\
b_{d-1,1} & b_{d-1,2} & \ldots & 0
\end{array}\right)\left(\begin{array}{c}
x_{1} \\
x_{2} \\
\vdots \\
x_{d-1}
\end{array}\right)
$$

$b_{i, j} \in I_{d}^{n-2}$. Therefore, $a \in I^{n-1}$ and $a x_{d}=\sum_{i, j \neq d}^{i \neq j} b_{i, j} x_{i} x_{j} \in\left(\sum_{1 \leq i<j \leq d-1} x_{i} x_{j} I_{d}^{n-2}\right)$. Conversely, take $a \in\left(\left(\sum_{1 \leq i<j \leq d-1} x_{i} x_{j} I_{d}^{n-2}\right): x_{d}\right) \cap I^{n-1}$. So there exist $b_{i, j} \in I_{d}^{n-2}$ such that $a x_{d}=x_{1} c_{1}+\cdots+x_{d-1} c_{d-1}$ where

$$
\left(\begin{array}{c}
c_{1} \\
c_{2} \\
\vdots \\
c_{d-1}
\end{array}\right)=\left(\begin{array}{cccc}
0 & b_{1,2} & \ldots & b_{1, d-1} \\
b_{2,1} & 0 & \ldots & b_{2, d-1} \\
\vdots & \vdots & \ddots & \vdots \\
b_{d-1,1} & b_{d-1,2} & \ldots & 0
\end{array}\right)\left(\begin{array}{c}
x_{1} \\
x_{2} \\
\vdots \\
x_{d-1}
\end{array}\right)
$$


Since $E(I)_{n}=0$, then (by Lemma 4.1)

$$
\begin{aligned}
\left(\begin{array}{c}
-c_{1} \\
-c_{2} \\
\vdots \\
-c_{d-1} \\
a
\end{array}\right)= & {\left[\left(\begin{array}{ccccc}
0 & 0 & \ldots & 0 & e_{1, d} \\
0 & 0 & \ldots & 0 & e_{2, d} \\
\vdots & \vdots & \ddots & \vdots & \vdots \\
0 & 0 & \ldots & 0 & e_{d-1, d} \\
-e_{1, d} & -e_{2, d} & \ldots & -e_{d-1, d} & 0
\end{array}\right)+\left(\begin{array}{ccccc}
0 & f_{1,2} & \ldots & f_{1, d-1} & 0 \\
-f_{1,2} & 0 & \ldots & f_{2, d-1} & 0 \\
\vdots & \vdots & \ddots & \vdots & \vdots \\
-f_{1, d-1} & -f_{2, d-1} & \ldots & 0 & 0 \\
0 & 0 & \ldots & 0 & 0
\end{array}\right)\right.} \\
+x_{d} & \left.\left(\begin{array}{ccccc}
0 & g_{1,2} & \ldots & g_{1, d-1} & 0 \\
-g_{1,2} & 0 & \ldots & g_{2, d-1} & 0 \\
\vdots & \vdots & \ddots & \vdots & \vdots \\
-g_{1, d-1} & -g_{2, d-1} & \ldots & 0 & 0 \\
0 & 0 & \ldots & 0 & 0
\end{array}\right)\right]\left(\begin{array}{c}
x_{1} \\
x_{2} \\
\vdots \\
x_{d-1} \\
x_{d}
\end{array}\right)
\end{aligned}
$$

where $e_{i, d} \in I^{n-2}, f_{i, j} \in I_{d}^{n-2}$ and $g_{i, j} \in I^{n-3}$. Put

$$
\left(\begin{array}{c}
h_{1} \\
h_{2} \\
\vdots \\
h_{d-1}
\end{array}\right)=\left(\begin{array}{c}
-e_{1, d} \\
-e_{2, d} \\
\vdots \\
-e_{d-1, d}
\end{array}\right)-\left(\begin{array}{cccc}
0 & g_{1,2} & \ldots & g_{1, d-1} \\
-g_{1,2} & 0 & \ldots & g_{2, d-1} \\
\vdots & \vdots & \ddots & \vdots \\
-g_{1, d-1} & -g_{2, d-1} & \ldots & 0
\end{array}\right)\left(\begin{array}{c}
x_{1} \\
x_{2} \\
\vdots \\
x_{d-1}
\end{array}\right)
$$

Then, $x_{1} h_{1}+\cdots+x_{d-1} h_{d-1}=a$ and $x_{d}\left(\begin{array}{c}h_{1} \\ \vdots \\ h_{d-1}\end{array}\right)=x_{d}\left(\begin{array}{c}-e_{1, d} \\ \vdots \\ -e_{d-1, d}\end{array}\right)-x_{d}\left(g_{i, j}\right)\left(\begin{array}{c}x_{1} \\ \vdots \\ x_{d-1}\end{array}\right)=$ $\left(\begin{array}{c}c_{1} \\ \vdots \\ c_{d-1}\end{array}\right)+\left(f_{i, j}\right)\left(\begin{array}{c}x_{1} \\ \vdots \\ x_{d-1}\end{array}\right)=\left(b_{i, j}+f_{i, j}\right)\left(\begin{array}{c}x_{1} \\ \vdots \\ x_{d-1}\end{array}\right)$. Thus, $a \in \sum_{i=1}^{d-1} x_{i}\left(\left(I_{i, d} I_{d}^{n-2}: x_{d}\right) \cap I^{n-2}\right)$.

Now, suppose that $(i),(i i)$ and $(i i i)$ hold and let us prove $E(I)_{n}=0$ by using Lemma 4.1. Take $\left(a_{1}, \ldots, a_{d}\right) \in\left(I^{n-1}\right)^{\oplus d}$ such that $a_{1} x_{1}+\cdots a_{d} x_{d}=0$. Fix $i \in\{1, \ldots, d\}$. Since $a_{i} \in I^{n-1}=A x_{i}^{n-1}+I_{i} I^{n-2}$, then $a_{i}=b_{i} x_{i}^{n-1}+\sum_{j \neq i} b_{j} x_{j}$ with $b_{i} \in A$ and $b_{j} \in I^{n-2}$. Since $0=\sum_{j=1}^{d} a_{j} x_{j}=b_{i} x_{i}^{n}+\sum_{j \neq i}\left(a_{j}+b_{j} x_{i}\right) x_{j}$, then $b_{i} \in I_{i} I^{n-1}: x_{i}^{n}=I_{i} I^{n-2}: x_{i}^{n-1}$ (by $\left.(i)\right)$. Thus $b_{i} x_{i}^{n-1}=\sum_{j \neq i} c_{j} x_{j}, c_{j} \in I^{n-2}$ and $a_{i}=\sum_{j \neq i}\left(b_{j}+c_{j}\right) x_{j} \in I_{i} I^{n-2}$. Hence, we can write $\left(\begin{array}{c}a_{1} \\ a_{2} \\ \vdots \\ a_{d}\end{array}\right)=\left(\begin{array}{cccc}0 & b_{1,2} & \ldots & b_{1, d} \\ b_{2,1} & 0 & \ldots & b_{2, d} \\ \vdots & \vdots & \ddots & \vdots \\ b_{d, 1} & b_{d, 2} & \ldots & 0\end{array}\right)\left(\begin{array}{c}x_{1} \\ x_{2} \\ \vdots \\ x_{d}\end{array}\right)$ where $b_{i, j} \in I^{n-2}$. For $i, j \neq d, i \neq j$, put $b_{i, j}=e_{i, j}+x_{d} h_{i, j}$ with $e_{i, j} \in I_{d}^{n-2}$ and $h_{i, j} \in I^{n-3}$. For $i \neq d$, put $c_{i, d}=b_{i, d}+\sum_{j \neq i, d} h_{i, j} x_{j}$. Then,

$$
\left(\begin{array}{c}
a_{1} \\
\vdots \\
a_{d-1} \\
a_{d}
\end{array}\right)=\left(\begin{array}{cccc}
0 & \ldots & 0 & c_{1, d} \\
\vdots & \ddots & \vdots & \vdots \\
0 & \ldots & 0 & c_{d-1, d} \\
b_{d, 1} & \ldots & b_{d, d-1} & 0
\end{array}\right)\left(\begin{array}{c}
x_{1} \\
\vdots \\
x_{d-1} \\
x_{d}
\end{array}\right)+\left(\begin{array}{cccc}
0 & \ldots & e_{1, d-1} & 0 \\
\vdots & \ddots & \vdots & \vdots \\
e_{d-1, d} & \ldots & 0 & 0 \\
0 & \ldots & 0 & 0
\end{array}\right)\left(\begin{array}{c}
x_{1} \\
\vdots \\
x_{d-1} \\
x_{d}
\end{array}\right)
$$

Since $\sum_{i=1}^{d} a_{i} x_{i}=0$, then $x_{d}\left(\sum_{i=1}^{d-1}\left(b_{d, i}+c_{i, d}\right) x_{i}\right)=-\sum_{i, j \neq d}^{i \neq j} x_{i} x_{j} e_{i, j} \in \sum_{1 \leq i<j \leq d-1} x_{i} x_{j} I_{d}^{n-2}$ and, by hypothesis $(i i),\left(b_{d, 1}+c_{1, d}\right) x_{1}+\cdots\left(b_{d, d-1}+c_{d-1, d}\right) x_{d-1}=f_{1} x_{1}+\cdots+f_{d-1} x_{d-1}$ 
where $f_{i} \in I^{n-2}$ and $x_{d}\left(\begin{array}{c}f_{1} \\ f_{2} \\ \vdots \\ f_{d-1}\end{array}\right)=\left(\begin{array}{cccc}0 & g_{1,2} & \ldots & g_{1, d-1} \\ g_{2,1} & 0 & \ldots & g_{2, d-1} \\ \vdots & \vdots & \ddots & \vdots \\ g_{d-1,1} & g_{d-1,2} & \ldots & 0\end{array}\right)\left(\begin{array}{c}x_{1} \\ x_{2} \\ \vdots \\ x_{d-1}\end{array}\right), g_{i, j} \in I_{d}^{n-2}$. Therefore,

$$
\left\{\begin{array}{cc}
a_{d}= & b_{d, 1} x_{1}+\cdots+b_{d, d-1} x_{d-1}=\left(f_{1}-c_{1, d}\right) x_{1}+\cdots+\left(f_{d-1}-c_{d-1, d}\right) x_{d-1} \\
a_{d-1}= & e_{d-1,1} x_{1}+\cdots+e_{d-1, d-2} x_{d-2}+c_{d-1, d} x_{d}= \\
& \left(e_{d-1,1}+g_{d-1,1}\right) x_{1}+\cdots+\left(e_{d-1, d-2}+g_{d-1, d-2}\right) x_{d-2}+\left(c_{d-1, d}-f_{d-1}\right) x_{d} \\
& \vdots \\
\vdots & \\
a_{1}= & e_{1,2} x_{2}+\cdots+e_{1, d-1} x_{d-1}+c_{1, d} x_{d}= \\
& \left(e_{1,2}+g_{1,2}\right) x_{2}+\cdots+\left(e_{1, d-1}+g_{1, d-1}\right) x_{d-1}+\left(c_{1, d}-f_{1}\right) x_{d}
\end{array}\right.
$$

We thus can write $\left(\begin{array}{c}a_{1} \\ a_{2} \\ \vdots \\ a_{d-1} \\ a_{d}\end{array}\right)=\left(\begin{array}{ccccc}0 & \tilde{c}_{1,2} & \ldots & \tilde{c}_{1, d-1} & \tilde{b}_{1, d} \\ \tilde{c}_{2,1} & 0 & \ldots & \tilde{c}_{2, d-1} & \tilde{b}_{2, d} \\ \vdots & \vdots & \ddots & \vdots & \vdots \\ \tilde{c}_{d-1,1} & \tilde{c}_{d-1,2} & \ldots & 0 & \tilde{b}_{d-1, d} \\ -\tilde{b}_{1, d} & -\tilde{b}_{2, d} & \ldots & -\tilde{b}_{d-1, d} & 0\end{array}\right)\left(\begin{array}{c}x_{1} \\ x_{2} \\ \vdots \\ x_{d-1} \\ x_{d}\end{array}\right)$, with $\tilde{b}_{i, d} \in$ $I^{n-2}$, but $\tilde{c}_{i, j} \in I_{d}^{n-2}$. Applying hypothesis $(i i i)$ to $\left(\begin{array}{cccc}0 & \tilde{c}_{1,2} & \ldots & \tilde{c}_{1, d-1} \\ \tilde{c}_{2,1} & 0 & \ldots & \tilde{c}_{2, d-1} \\ \vdots & \vdots & \ddots & \vdots \\ \tilde{c}_{d-1,1} & \tilde{c}_{d-1,2} & \ldots & 0\end{array}\right)\left(\begin{array}{c}x_{1} \\ x_{2} \\ \vdots \\ x_{d-1}\end{array}\right)$, we finish.

Corollary 4.8 Induction Theorem Let $I$ be generated by $d$ elements $x_{1}, \ldots, x_{d}(d \geq 3)$ and take $n \geq 2$. Suppose that $E\left(I_{d}\right)_{n}=0$. Then $E(I)_{n}=0$ if, and only if,

(i) $I_{i} I^{n-1}: x_{i}^{n}=I_{i} I^{n-2}: x_{i}^{n-1}$ for all $i=1, \ldots, d$,

(ii) $\left(\left(\sum_{1 \leq i<j \leq d-1} x_{i} x_{j} I_{d}^{n-2}\right): x_{d}\right) \cap I^{n-1}=\sum_{i=1}^{d-1} x_{i}\left(\left(I_{i, d} I_{d}^{n-2}: x_{d}\right) \cap I^{n-2}\right)$.

Proof. By lemma 4.1, $E\left(I_{d}\right)_{n}=0$ assures that condition $(i i i)$ of Theorem 4.7 is fulfilled.

Corollary 4.9 Let $\underline{x}=x_{1}, \ldots, x_{d}$ be d elements of $A$. Then, $\underline{x}$ is a sequence of linear type if, and only if, for all $n \geq 2$

(i) $\left(x_{1}, \ldots, \widehat{x}_{i}, \ldots, x_{k}\right)\left(x_{1}, \ldots, x_{k}\right)^{n-1}: x_{i}^{n}=\left(x_{1}, \ldots, \widehat{x}_{i}, \ldots, x_{k}\right)\left(x_{1}, \ldots, x_{k}\right)^{n-2}: x_{i}^{n-1}$ for all $1 \leq i \leq k \leq d$,

(ii) For all $1 \leq i<j<k \leq d$,

$$
\begin{aligned}
& \left(\left(\sum_{1 \leq i<j \leq k-1} x_{i} x_{j}\left(x_{1}, \ldots, x_{k-1}\right)^{n-2}\right): x_{k}\right) \cap\left(x_{1}, \ldots, x_{k}\right)^{n-1}= \\
& \sum_{i=1}^{k-1} x_{i}\left(\left(\left(x_{1}, \ldots, \widehat{x}_{i}, \ldots, x_{k-1}\right)\left(x_{1}, \ldots, x_{k-1}\right)^{n-2}: x_{k}\right) \cap\left(x_{1}, \ldots, x_{k}\right)^{n-2}\right),
\end{aligned}
$$

(understanding $\sum_{1 \leq i<j \leq k-1}(\ldots)=0$ for $k \leq 2$ and $\sum_{i=1}^{k-1}(\ldots)=0$ for $k=1$ ). 
Remark 4.10 With the hypothesis $E\left(I_{d}\right)_{n}=0$ of Corollary 4.8, it is not hard to prove that $E(I)_{n}=0$ is equivalent to

(i) $I_{d} I^{n-1}: x_{d}^{n}=I_{d} I^{n-2}: x_{d}^{n-1}$,

(ii) If $\left(a_{1}, \ldots, a_{d-1}\right) \in\left(I^{n-1}\right)^{\oplus(d-1)}$ with $a_{1} x_{1}+\ldots+a_{d-1} x_{d-1}=0$, then there exists $\left(b_{1}, \ldots, b_{d-1}\right) \in\left(I^{n-2}\right)^{\oplus(d-1)}$ and $\left(c_{1}, \ldots, c_{d-1}\right) \in\left(I_{d}^{n-1}\right)^{\oplus(d-1)}$ such that $b_{1} x_{1}+\ldots+$ $b_{d-1} x_{d-1}=0$ and $a_{i}=x_{d} b_{i}+c_{i}$ for all $i=1, \ldots, d-1$.

In fact, this is the expected generalization of Costa's Induction Theorem (see 4 of [6]).

Corollary 4.11 Let I be generated by $x_{1}, x_{2}, x_{3}$ and take $n \geq 2$. Then, $E(I)_{n}=0$ if, and only if,

(i) $I_{i} I^{n-1}: x_{i}^{n}=I_{i} I^{n-2}: x_{i}^{n-1}$ for all $i=1,2,3$,

(ii) $\left(x_{1} x_{2} I_{3}^{n-2}: x_{3}\right) \cap I^{n-1}=x_{1}\left(\left(x_{2} I_{3}^{n-2}: x_{3}\right) \cap I^{n-2}\right)+x_{2}\left(\left(x_{1} I_{3}^{n-2}: x_{3}\right) \cap I^{n-2}\right)$,

(iii) $\left(0: x_{1}\right) \cap I^{n-1}=\left\{a_{2} x_{2}+a_{3} x_{3} \mid a_{i} \in I^{n-2}, a_{2} x_{1}=b x_{3}, a_{3} x_{1}=-b x_{2}\right.$ for $\left.b \in I_{1}^{n-2}\right\}$.

Moreover, if $\left(0: x_{1} x_{2}\right) \cap I_{3}^{n-2}=\left(0: x_{1}\right) \cap I_{3}^{n-2}+\left(0: x_{2}\right) \cap I_{3}^{n-2}$ (for instance, if $\left.E\left(I_{3}\right)_{n}=0\right)$ then condition (iii) can be skipped.

Proof. Suppose $E(I)_{n}=0$. Then, Lemma 4.2 assures $(i)$, Lemma 4.4 assures (iii) and Theorem 4.7 assures (ii). Conversely, suppose (i), (ii) and (iii) hold and let us prove $E(I)_{n}=0$ by proving $(i i i)$ of Theorem 4.7. So take $\left(\begin{array}{c}a_{1} \\ a_{2}\end{array}\right)=\left(\begin{array}{ll}0 & b \\ c & 0\end{array}\right)\left(\begin{array}{l}x_{1} \\ x_{2}\end{array}\right)$ with $a_{1} x_{1}+a_{2} x_{2}=0$ and $b, c \in I_{3}^{n-2}$. Since $(b+c) x_{1} x_{2}=0$, then $(b+c) x_{2} \in\left(0: x_{1}\right) \cap I^{n-1}$ and, by hypothesis $(i i i),(b+c) x_{2}=e x_{2}+f x_{3}$ with $e, f \in I^{n-2}$ and $e x_{1}=g x_{3}, f x_{1}=-g x_{2}$ for some $g \in I_{1}^{n-2}$. Thus, $\left(\begin{array}{c}a_{1} \\ a_{2} \\ 0\end{array}\right)=\left(\begin{array}{ccc}0 & e-c & -f \\ c-e & 0 & g \\ f & -g & 0\end{array}\right)\left(\begin{array}{c}x_{1} \\ x_{2} \\ x_{3}\end{array}\right)$. Analogously, one could prove that $\left(0: x_{1} x_{2}\right) \cap I_{3}^{n-2}=\left(0: x_{1}\right) \cap I_{3}^{n-2}+\left(0: x_{2}\right) \cap I_{3}^{n-2}$ implies (iii) of 4.7.

ACKNOWLEDGEMENTS: I am very grateful to J.M. Giral and S. Zarzuela for the very helpful discussions concerning this paper.

\section{References}

[1] M. André. Homologie des algèbres commutatives. Grundlehren no. 206 (Springer-Verlag, 1974).

[2] J. Backelin and R. Fröberg. Koszul algebras, Veronese subrings and rings with linear resolutions. Rev. Roumaine Math. Pure Appl. 30 (1985), 85-97.

[3] W. Bruns and J. Herzog. Cohen-Macaulay rings. Cambridge Studies in Advanced Math. no. 39 (Cambridge University Press, 1993).

[5] D. L. Costa. On the Torsion-Freeness of the Symmetric Powers of an Ideal. J. Algebra 80 (1983), 152-158. 
[6] D. L. Costa. Sequences of linear type. J. Algebra 94 (1985), 256-263.

[7] B. Johnston and D. Katz. On the relation type of large powers of an ideal. Mathematika 41 (1994), 209-214.

[8] J. Herzog, A. Simis and W. V. Vasconcelos. Koszul homology and blowing-up rings. Lecture Notes in Pure and Applied Math. vol. 84 (Marcel Dekker Inc., 1982), pp. 79-169.

[9] M. Kühl. On the symmetric algebra of an ideal. Manuscripta Math. 37 (1982), 49-60.

[10] A. Micali and N. Roby. Algèbres symétriques et syzygies. J. Algebra 17 (1971), 460-469.

[11] F. Planas Vilanova. Sur l'annulation du deuxième foncteur de (co)homologie d'André-Quillen. Manuscripta Math. 87 (1995), 349-357.

[12] F. Planas Vilanova. On the vanishing and non-rigidity of the André-Quillen (co)homology. $J$. Pure and Appl. Alg. (To appear).

[13] P. Schenzel. Castelnuovo's index of regularity and reduction numbers. Banach Center Publications 26 (2) (1990), 201-208.

[14] G. Valla. On the symmetric and Rees algebra of an ideal. Manuscripta Math. 30 (1980), 239-255. 